\title{
Awake single-access (uniportal) video-assisted thoracoscopic surgery for spontaneous pneumothorax
}

\author{
Gaetano Rocco, MD, FRCSEd, Antonello La Rocca, MD, Nicola Martucci, MD, and \\ Rosanna Accardo, MD, Naples, Italy
}

According to the currently available literature, consensus exists as to the management of bilateral pneumothorax

From the Department of Thoracic Surgery and Oncology and the Service of Anesthesiology, National Cancer Institute, Pascale Foundation, Naples, Italy.

Disclosures: Authors have nothing to disclose with regard to commercial support.

Received for publication Nov 4, 2010; revisions received Dec 24, 2010; accepted for publication Jan 24, 2011; available ahead of print March 7, 2011.

Address for reprints: Gaetano Rocco, MD, FRCSEd, Via Terminio 1, Serino (Avel-

lino) 83028 Italy (E-mail: Gaetano.Rocco@btopenworld.com).

J Thorac Cardiovasc Surg 2011;142:944-5

$0022-5223 / \$ 36.00$

Copyright (c) 2011 by The American Association for Thoracic Surgery

doi:10.1016/j.jtcvs.2011.01.048

through minimally invasive VATS. $^{1} \quad$ Single-access (uniportal) video-assisted thoracoscopic surgery (VATS) can make the surgical option suitable also after the first episode of primary spontaneous pneumothorax, because it does not entail additional incisions beyond the one already in use for the chest drain. ${ }^{2,3}$ In light of our preliminary experience with uniportal VATS wedge resection in the awake patient, ${ }^{4}$ we also tested this approach in the case of a patient with bilateral apical bullae.

\section{CLINICAL SUMMARY}

A 23-year-old male patient had a recurrent spontaneous pneumothorax develop, prompting a chest computed tomographic scan. The scan demonstrated bilateral apical bullae. With general anesthesia and single-lung ventilation, the patient underwent single-access (uniportal)

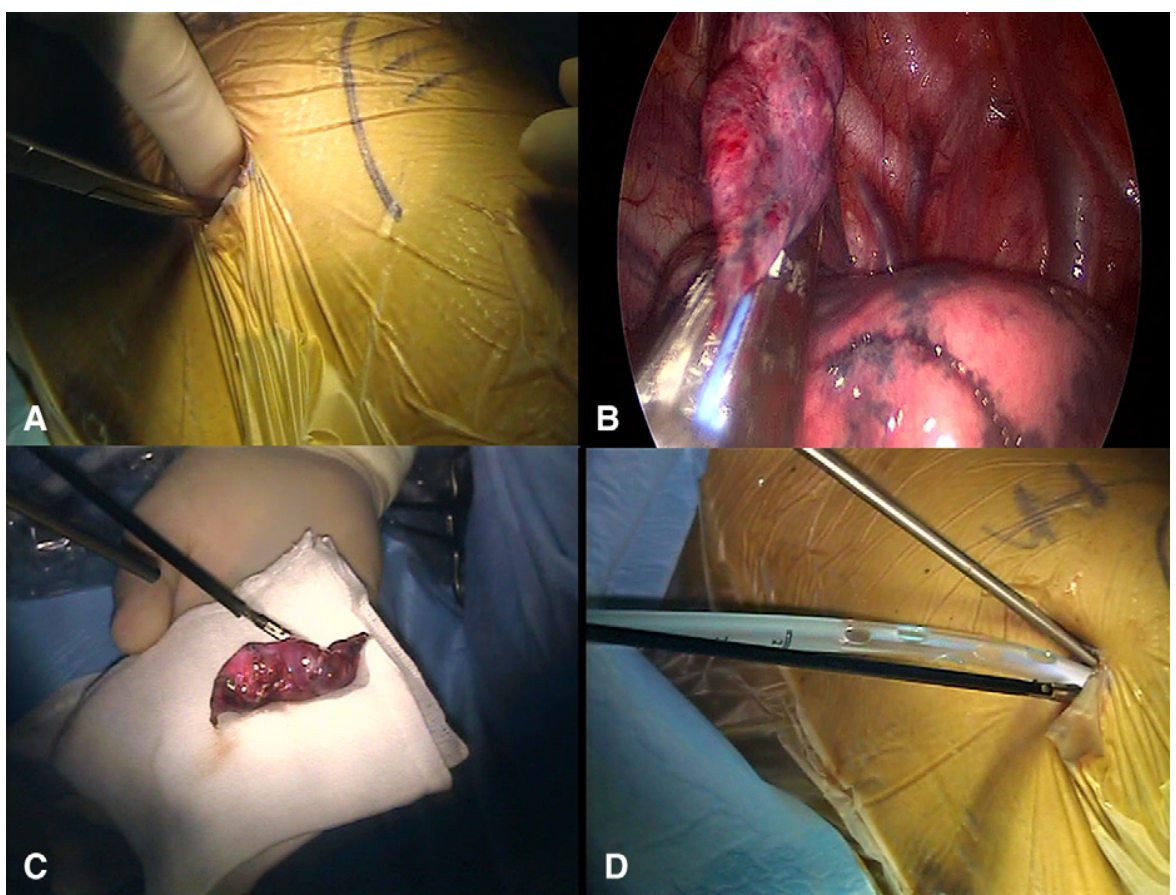

FIGURE 1. A, Close-up view of the single thoracoscopic incision to the right chest measuring about $2 \mathrm{~cm}$ (1 finger breadth). B, Intraoperative view demonstrating the resection of apical bullous disease according to a sagittal plane, as per the uniportal video-assisted thoracoscopic surgical approach. ${ }^{2} \mathrm{C}$ and $\mathrm{D}$, The specimen is removed, and the chest drain inserted through the same operative incision under thoracoscopic guidance after talc poudrage. 
VATS and resection of the left upper lobe apex, along with apical pleural abrasion and talc poudrage ( $2 \mathrm{~g}$ Steritalc; Novatech New Biotechnology for Life, La Ciotat, France). At 1-month follow-up, the left lung was completely expanded on chest radiography. When 7 months later the patient had development of a right-sided pneumothorax, he was offered an awake uniportal VATS wedge resection of the right upper lobe apex. ${ }^{4}$ In brief, with the patient under mild sedation (4 mg midazolam intravenously and 0.5 $\mathrm{mg} /[\mathrm{kg} \cdot \mathrm{h}]$ propofol), epidural anesthesia was administered by single-shot injection of ropivacaine hydrochloride (INN ropivacaine) at the T5-6 level to ensure adequate analgesia for the uniportal procedure and in case of conversion to conventional VATS or minithoracotomy while proceeding to endotracheal intubation. Supplemental oxygen through nasal prongs was also provided to maintain arterial oxygen saturation greater than $90 \%$. Subsequently, a 3F Foley balloon catheter was inserted bronchoscopically and then inflated to facilitate right upper lobe collapse. A single $2.5-\mathrm{cm}$ incision was placed in the third interspace along the right midaxillary line (Figure 1, A, Video 1), and the bullae were resected according to the usual technique ${ }^{2}$ (Figure $1, B$ ). In brief, an articulating endograsper was introduced through the single incision parallel to the $5-\mathrm{mm}, 0^{\circ}$ videothoracoscope. By deploying the articulating arm, the target bullae were grasped suspended along a sagittal, craniocaudal plane. An articulating endostapler loaded with a 45-mm blue cartridge was inserted parallel to the grasper-thoracoscope ensemble, taking advantage of the full laterality of the incision. The endostapler must be articulated outside the chest and then inserted as a mediastinoscope under the pretracheal fascia. The endograsper gently accommodated the parenchyma to be resected in between the endostapler jaws. To complete the resection, multiple firings could be needed while pleurodesis was ensured through talc poudrage ( 2 g Steritalc; Figure 1, $C$ and $D$ ). The patient was observed overnight and was discharged the next morning with a flutter bag, which was removed the next day in the outpatient clinic. At 1 year-follow up, neither signs nor symptoms of recurrent pneumothorax were observed on either side.

\section{DISCUSSION}

Relative to conventional 3-port VATS, uniportal VATS for pneumothorax appears to reduce postoperative pain, paresthesia, and hospital stay. ${ }^{3,4}$ An obvious advantage of uniportal VATS relative to medical thoracoscopyintended as a thoracoscopic procedure performed with local anesthesia usually to diagnose pleural effusions or to effect chemical pleurodesis for pneumothorax-is the possibility of removing diseased parenchyma and proceeding to either mechanical or chemical pleurodesis. ${ }^{2,5}$ Controversy still exists regarding the management of first episodes of primary spontaneous pneumothorax. An immediate surgical approach by uniportal VATS after the first presentation of primary spontaneous pneumothorax may be facilitated by the resort to locoregional anesthesia in the awake patient. ${ }^{1,4}$ The absence of general anesthesia and double-lung ventilation and the reduction of hospital costs could decisively shift the balance in the management of spontaneous pneumothorax toward VATS. ${ }^{4}$ In fact, this surgical procedure can even be done in an outpatient setting, thereby minimizing the need for a dedicated hospital suite and beds. ${ }^{4}$

\section{References}

1. Chou SH, Li HP, Lee JY, Chang SJ, Lee YL, Chang YT, et al. Is prophylactic treatment of contralateral blebs in patients with primary spontaneous pneumothorax indicated? J Thorac Cardiovasc Surg. 2010;139:1241-5.

2. Rocco G, Martin-Ucar A, Passera E. Uniportal VATS wedge pulmonary resections. Ann Thorac Surg. 2004;77:726-8.

3. Jutley RS, Khalil MW, Rocco G. Uniportal vs standard three-port VATS technique for spontaneous pneumothorax: comparison of post-operative pain and residual paraesthesia. Eur J Cardiothorac Surg. 2005;28:43-6.

4. Rocco G, Romano V, Accardo R, Tempesta A, La Manna C, La Rocca A, et al Awake single-access (uniportal) video-assisted thoracoscopic surgery for peripheral pulmonary nodules in a complete ambulatory setting. Ann Thorac Surg. 2010; 89:1625-7.

5. Cardillo G, Carleo F, Giunti R, Carbone L, Mariotta S, Salvadori L, et al Videothoracoscopic talc poudrage in primary spontaneous pneumothorax: a single-institution experience in 861 cases. J Thorac Cardiovasc Surg. 2006;131: 322-8. 